\title{
Array Antenna Design for Passive Coherent Location Systems with Non-Uniform Array Configurations
}

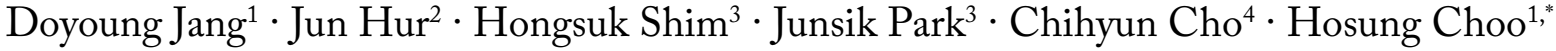

\begin{abstract}
This study investigates non-uniform array configurations to maximize the beamforming performance of passive coherent location (PCL) systems. The proposed array consists of eight dipole elements that are divided into two groups with different distances from the array center. This double-layered non-uniform configuration is designed considering the array antenna characteristics (peak-to-side lobe ratio, first null bandwidth, null width, and null depth). The resulting antenna array can provide more appropriate patterns for PCL systems than a conventional uniform circular array. The target detection performance of the proposed array in PCL systems is tested in a certain scenario. The results demonstrate that the proposed antenna in PCL systems can detect the target with Doppler and range errors of $1 \mathrm{~Hz}$ and $1.2 \mathrm{~km}$, respectively, in a given situation.
\end{abstract}

Key Words: Antenna, Array Antenna, Passive Coherent Location, Passive Radar.

\section{INTRODUCTION}

Passive coherent location (PCL) systems based on noncooperative sources such as FM and TV signals have been developed successfully in recent years [1-4]. The purpose of a PCL system is to detect and track airborne targets using a reference and a surveillance channel. The reference channel, used to obtain commercial broadcast signals, should steer the beam toward a base station, and at the same time the surveillance channel should have a radiation pattern that includes deep null toward the base station. From the correlation between the signals of the two channels, targets can be detected and tracked $[5,6]$. However, in general, echo signals reflected from potential targets are extremely weak and are obscured by strong direct sig- nals. To increase the detectability of echo signals by minimizing the terrestrial multipath effect and other clutters, interference suppression techniques that support the accuracy of directionof-arrival information are typically used [7-10]. In addition, the probability hypothesis density and the multi-frame assignment have been proposed to enhance the detection performance of PCL systems [11-13]. Their performance can be further optimized by improving the performance of the antennas employed in PCL systems. For example, antenna elements with multiband and dual polarization characteristics have been designed to increase the probability of target detection by expanding the operating frequency and available polarization. Although studies aiming to determine the optimal beam pattern of the array for PCL systems according to various array configurations have also

Manuscript received February 3, 2020 ; Revised March 18, 2020 ; Accepted March 30, 2020. (ID No. 20200203-014J)

${ }^{1}$ School of Electronic and Electrical Engineering, Hongik University, Seoul, Korea.

${ }^{2}$ Metamaterial Electronic Device Research Center, Hongik University, Seoul, Korea.

${ }^{3}$ Hanwha Systems Co. Ltd., Seongnam, Korea.

${ }^{4}$ Korea Research Institute of Standards and Science, Daejeon, Korea.

"Corresponding Author: Hosung Choo (e-mail: hschoo@hongik.ac.kr)

This is an Open-Access article distributed under the terms of the Creative Commons Attribution Non-Commercial License (http://creativecommons.org/licenses/by-nc/4.0) which permits unrestricted non-commercial use, distribution, and reproduction in any medium, provided the original work is properly cited.

(c) Copyright The Korean Institute of Electromagnetic Engineering and Science. All Rights Reserved. 
been conducted $[14,15]$, they are limited to certain canonical forms of array configurations. Other studies have performed optimizations of array configurations using various optimization methods, such as the particle swarm algorithm [16], the invasive weed algorithm [17], and the genetic algorithm [18]. However, non-uniform array configurations that can effectively improve the array performance of PCL systems have not been sufficiently considered.

In this paper, we propose a non-uniform array configuration to maximize the beamforming performance of PCL systems. The proposed array consists of eight dipole elements divide into two groups with different distances from the array center. This double-layered non-uniform configuration is used to observe the trends of beamforming performance for various array configurations. Each element is designed and fabricated to operate in the FM frequency band, and the antenna performance required for PCL systems are examined. The array antenna characteristics - peak-to-side lobe ratio (PSLR), first null bandwidth (FNBW), null width, and null depth-are then investigated to derive the optimum array configuration. Parametric studies of various array distances are conducted, and the optimal configuration is determined by evaluating the cost function of the beamforming performance. To verify the performance enhancement of the proposed array, the reference and surveillance beams are compared with the conventional uniform circular array (UCA). The results demonstrate that the proposed nonuniform array configuration has more suitable beam patterns compared with the conventional UCA. The target detection performance of the proposed array for PCL systems is tested in a certain scenario.

\section{DESIGN OF THE DiPOLE ELEMENT}

Fig. 1 shows a photograph of the fabricated dipole element with a broadband balun (ADT1.5-1+, Mini-Circuits) embedded in the circuit board. The balun operates in a range from 1 to $300 \mathrm{MHz}$ with an insertion loss of less than $1 \mathrm{~dB}$. This structure can help minimize the pattern distortion by the unbalanced current of the coaxial feed while maintaining the broadband matching characteristics. Thanks to its compact size $(7.9 \times 5.6 \times$ $\left.2.8 \mathrm{~mm}^{3}\right)$, the balun can be mounted on a board, as shown in Fig. 1(a) and (b). It provides the housing structure with the circuit board to protect the circuit from external physical shocks while reliably attaching the dipole antenna to it. The fully manufactured dipole with the housing is shown in Fig. 1(c). The length and radius of the dipole are $b=134 \mathrm{~cm}$ and $r=7.5 \mathrm{~mm}$, respectively.

Fig. 2 provides a comparison of the measured and simulated voltage standing wave ratios (VSWRs) of the proposed dipole element (solid and dashed lines, respectively). The measurement

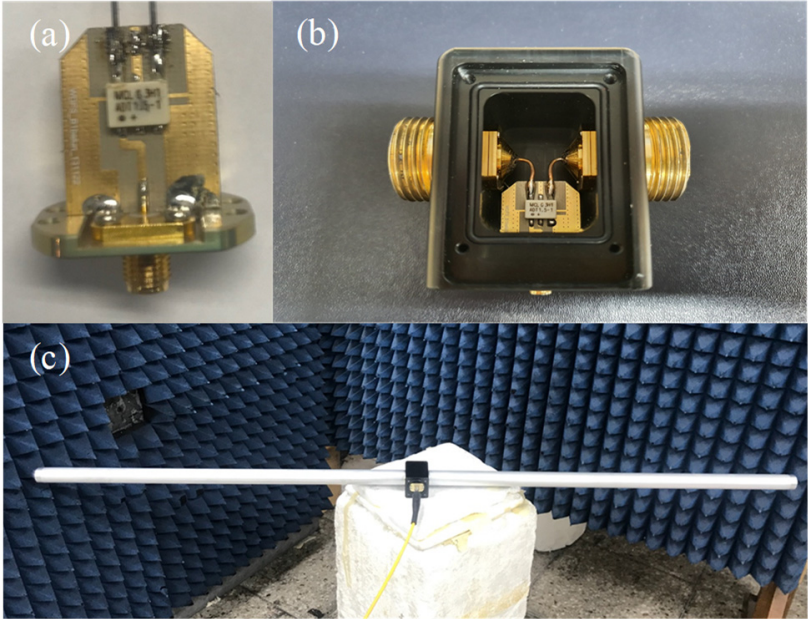

Fig. 1. Photograph of the dipole element for the PCL array: (a) balun with circuit board, (b) housing structure, and (c) dipole element.

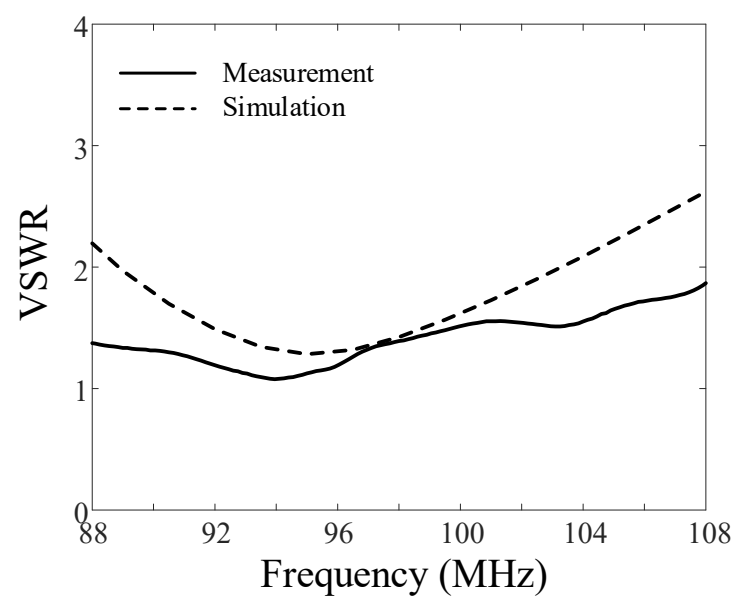

Fig. 2. Measured and simulated VSWRs of the dipole element.

is performed in a semi-anechoic chamber, resulting in a bandwidth of $23 \mathrm{MHz}$ (from $85 \mathrm{MHz}$ to $108 \mathrm{MHz}$, VSWR <2) with a fractional bandwidth greater than $23 \%$. The fractional bandwidth of the simulated VSWR is $17 \%$, which shows good agreement with the measured data. Fig. 3 illustrates the boresight gain as a function of frequency. The solid line represents the measured data, which maintains a gain above $1 \mathrm{dBi}$ from 88 to $108 \mathrm{MHz}$. The simulation values are similar to the measured values, with differences less than $0.5 \mathrm{~dB}$. These results show that the performance of the designed dipole element is suitable for a PCL system.

\section{PROPOSED ARRAY CONFIGURATION}

Fig. 4 shows the geometry of the proposed non-uniform eight-element array for PCL systems. It consists of two groups (inner and outer layers) with different distances $d_{1}$ and $d_{2}$ from the array center, as shown in Fig. 4(a). The expandable mast 


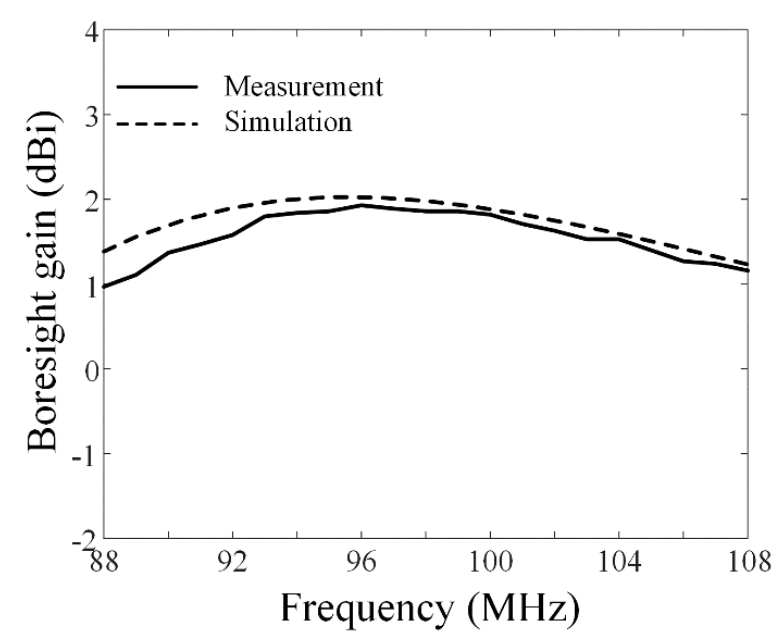

Fig. 3. Measured and simulated boresight gain of the dipole element.

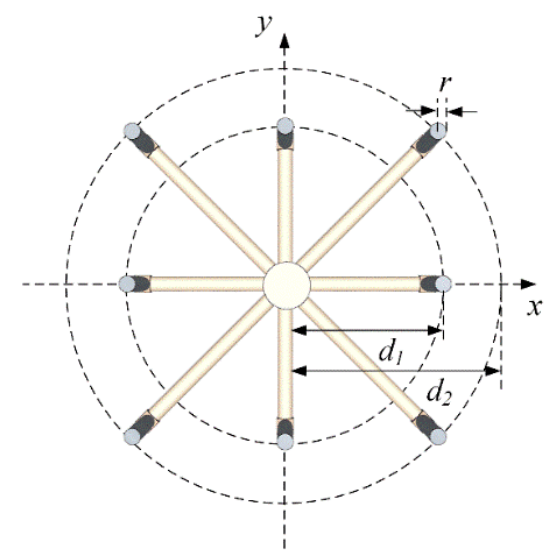

(a)

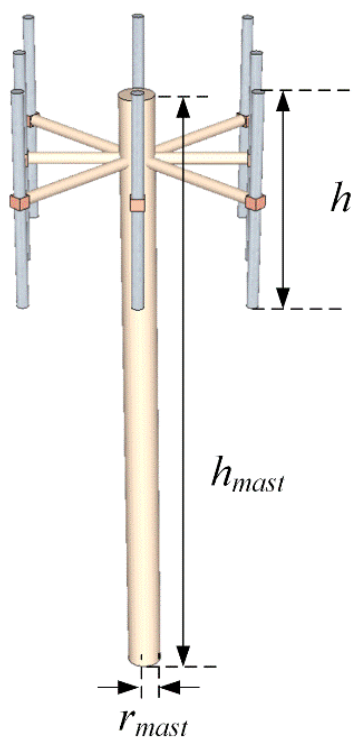

(b)

Fig. 4. Configuration of the proposed array: (a) top view and (b) isometric view.
Table 1. Parameters of the proposed array

\begin{tabular}{lc}
\hline Parameter & Value \\
\hline$d_{1}$ & $0.41 \lambda$ \\
$d_{2}$ & $0.32 \lambda$ \\
$d_{\text {ratio }}\left(d_{2} / d_{1}\right)$ & 0.8 \\
$b$ & $134 \mathrm{~cm}$ \\
$r$ & $7.5 \mathrm{~mm}$ \\
$h_{\text {mast }}$ & $8.6 \mathrm{~mm}$ \\
$r_{\text {mast }}$ & $30 \mathrm{~mm}$ \\
\hline
\end{tabular}

with length $h_{\text {mast }}$ and radius $r_{\text {mast }}$ is used to mount the array elements. WIPL-D Pro electromagnetic simulation software (WIPL-D, Belgrade, Serbia) is used to optimize and analyze the proposed configuration. The optimum design parameters are displayed in Table 1.

To effectively detect targets, the array antenna of the PCL system must be able to generate two different channels: the reference and the surveillance channel. Since PCL systems are passive radar systems that do not generate a reference signal, commercial broadcast signals from FM base stations are often used as reference signals for detecting targets. Therefore, the beam pattern of the reference channel should have a higher PSLR and narrower FNBW to obtain only the reference signal from the base station. However, the beam pattern of the surveillance channel requires a greater null depth and a narrower null width toward the base station. This is because the surveillance channel should only receive scattered signals from targets without the reference signals. The optimum array configuration is derived by considering the beam patterns of the reference and surveillance channels. The mask functions for the reference and surveillance beams are used to obtain the optimized beam patterns [15]. The mask function $F(\phi)$ can be calculated as

$$
A \cdot \omega=F(\phi)
$$

where $A$ and $\omega$ are the array manifold and weightings to create the beam pattern of the proposed non-uniform array, respectively. The weightings for the beam pattern are then calculated with the following equation:

$$
\omega=\left(A^{H} \cdot A\right)^{-1} \cdot A^{H} \cdot F(\phi)
$$

From Eq. (2), we can derive the weightings $\omega$ that can minimize the square error between the pattern and the mask function. Since the FM band ranges from 88 to $108 \mathrm{MHz}$, the array characteristics of the PSLR, FNBW, null depth, and null width are examined at three specific frequencies: 88, 98, and 108 $\mathrm{MHz}$. The cost function to assess array performance is defined to achieve the optimal array configuration as follows: 


$$
\begin{aligned}
& \operatorname{Cost}_{r}=\left(1-\alpha \frac{P S L R(x, y)}{P S L R_{\max }}\right)+\gamma \frac{F N B W(x, y)}{F N B W_{\max }} \\
& \text { Cost }_{s}=\left(1-\beta \frac{\text { Nulldepth }(x, y)}{\text { Nulldepth }_{\max }}\right)+\delta \frac{\text { Nullwidth }(x, y)_{\text {Nullwidth }_{\max }}}{\text { N }} \\
& \operatorname{Cost}_{\text {total }}=\text { Cost }_{r}+\text { Cost }_{s}
\end{aligned}
$$

where Cost $t_{r}$ and Cost $t_{s}$ are the cost functions of the reference and surveillance beams, respectively. These costs are computed using the PSLR, FNBW, null depth, and null width. Weight constants of $\alpha=0.8, \beta=0.5, \gamma=0.2$, and $\delta=0.5$ are used in the optimization. Lower cost means more appropriate beam pattern.

Fig. 5 illustrates the total cost map according to $d_{1}$ and $d_{\text {ratio. }}$. $d_{1}$ is changed from $0.4 \lambda$ to $0.6 \lambda$ at intervals of $0.01 \lambda$, and $d_{\text {ratio }}$ is varied from 0.5 to 1.5 with intervals of 0.05 . The minimum cost of 0.67 is obtained when $d_{1}$ and $d_{\text {ratio }}$ are $0.41 \lambda$ and 0.8 , respectively, while a cost of 0.7 is observed with the UCA configuration $\left(d_{1}=d_{2}=0.5 \lambda\right)$.

Fig. 6 shows the patterns of the reference and surveillance beams at a center frequency of $98 \mathrm{MHz}$ in the FM band. The solid lines indicate the beam pattern of the proposed array, and the dashed lines represent the beam pattern of the UCA configuration. For the proposed array, the PSLR and FNBW are 13.3 $\mathrm{dB}$ and $139^{\circ}$, respectively, and the null depth and null width are $37.5 \mathrm{~dB}$ and $23^{\circ}$, respectively. The exact values of the PSLR, FNBW, null depth, and null width at all analysis frequencies are listed in Table 2. The results indicate that the average PSLR and null depth at three frequencies $(88,98$, and $108 \mathrm{MHz})$ are greater than those in the UCA configuration by $0.7 \mathrm{~dB}$ and 0.7 $\mathrm{dB}$, respectively.

To verify the detection performance using the proposed array, an amplitude range-Doppler map is created as shown Fig. 7. In the given scenario, the distance between the PCL radar and the target is assumed to be $100 \mathrm{~km}$, and the target's velocity is assumed to be $551 \mathrm{~m} / \mathrm{s}$. The received reference and surveillance

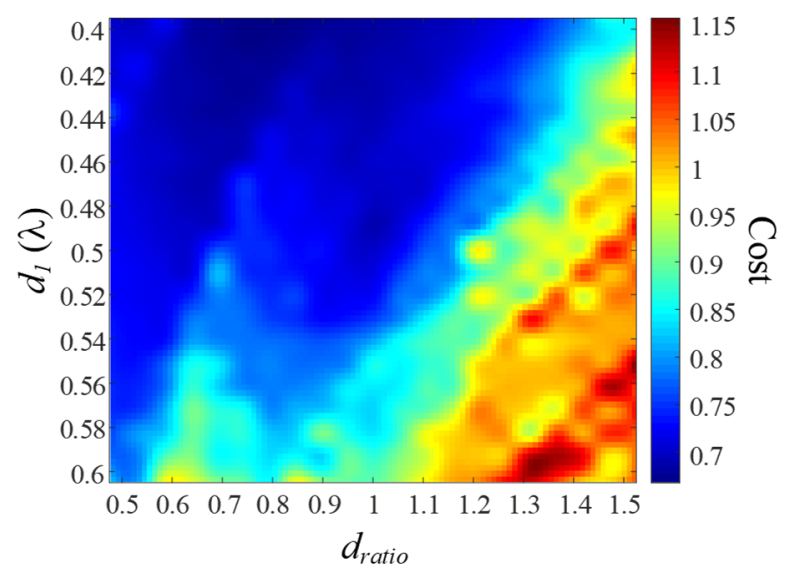

Fig. 5. Total cost map according to $d_{1}$ and $d_{\text {ratio. }}$

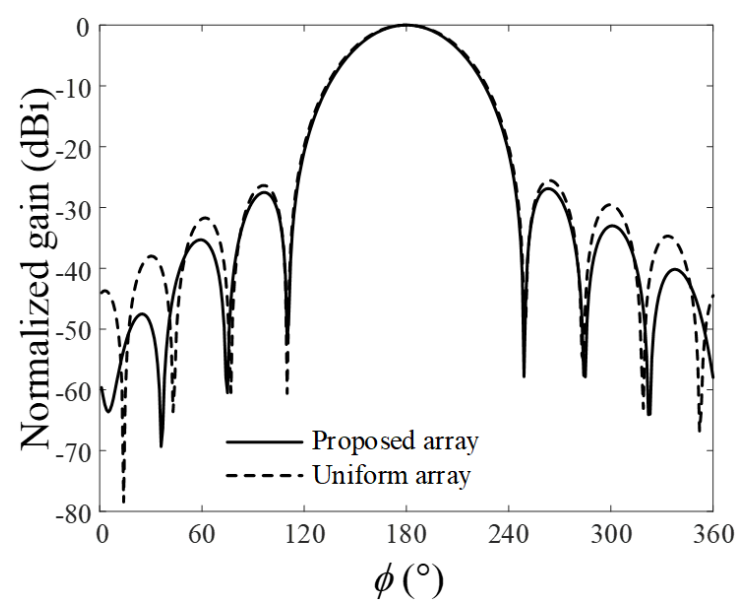

(a)

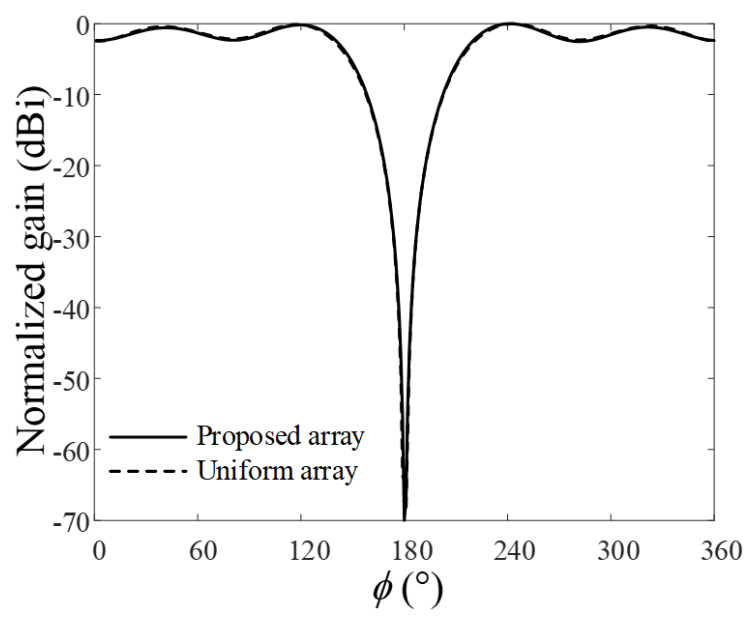

(b)

Fig. 6. Beam patterns of the proposed and uniform circular array at $98 \mathrm{MHz}$ : (a) reference channel and (b) surveillance channel.

signals are used in the cross-correlation equation to obtain the amplitude range-Doppler map. The cross correlation $\chi$ can be calculated as

$$
\chi\left(t, f_{d}\right)=\int_{-\infty}^{\infty} x(t) \cdot x(t-\tau)^{*} e^{j 2 \pi f_{d} t} \cdot d t
$$

Table 2. Comparison of array antenna characteristics

\begin{tabular}{lcccc}
\hline Parameter & $\begin{array}{c}\text { PSLR } \\
(\mathrm{dB})\end{array}$ & $\begin{array}{c}\text { FNBW } \\
\left({ }^{\circ}\right)\end{array}$ & $\begin{array}{c}\text { Null } \\
\text { depth } \\
(\mathrm{dB})\end{array}$ & $\begin{array}{c}\text { Null } \\
\text { width } \\
\left({ }^{\circ}\right)\end{array}$ \\
\hline $\begin{array}{l}\text { 88 MHz } \\
\text { Proposed array }\end{array}$ & 13.5 & 139 & 35.0 & 23 \\
$\quad 12.8$ & 140 & 32.1 & 23 \\
UCA & & & & \\
98 MHz & 13.3 & 139 & 37.5 & 23 \\
Proposed array & 12.5 & 140 & 38.0 & 23 \\
UCA & & & & \\
108 MHz & 12.9 & 139 & 32.4 & 27 \\
$\quad$ Proposed array & 12.3 & 140 & 32.8 & 23 \\
UCA & & & &
\end{tabular}




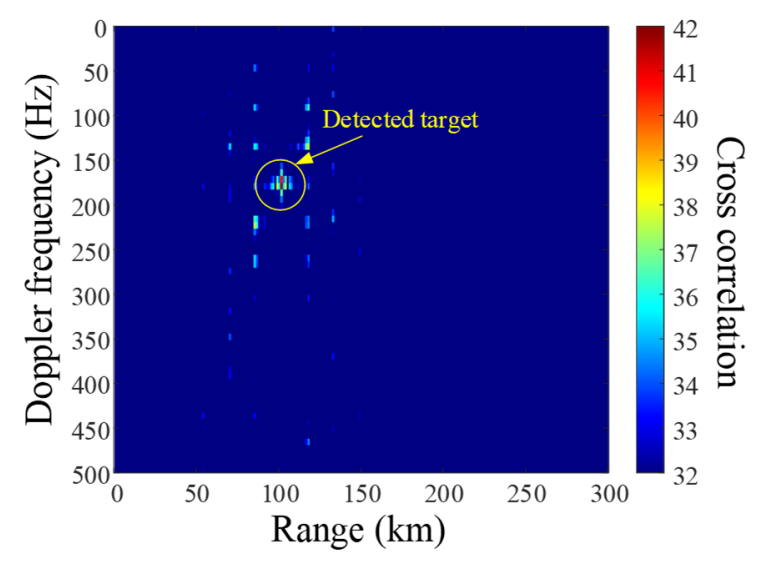

Fig. 7. Amplitude range-Doppler map for a detected target in a given scenario.

where $\tau$ is the signal delay between the received reference and surveillance signals, and $f_{d}$ is the Doppler frequency according to the target's velocity. With a target velocity of $551 \mathrm{~m} / \mathrm{s}$, the actual Doppler frequency is $180 \mathrm{~Hz}$. The value derived from the correlation equation is $179 \mathrm{~Hz}$. The estimated range between the PCL radar and the target is $101.2 \mathrm{~km}$, which is close to the scenario's range of $100 \mathrm{~km}$. These results demonstrate that the proposed non-uniform array for PCL systems can effectively detect targets.

\section{CONCLUSION}

Non-uniform array configurations were investigated to maximize the beamforming performance of PCL systems. The proposed array consisted of eight dipole elements that are divided into two groups with distances of $0.41 \lambda$ and $0.32 \lambda$ from the array center, respectively. The individual dipole element had a bandwidth of $23 \mathrm{MHz}$ (85 to $108 \mathrm{MHz}$, VSWR <2), which showed suitable performance for PCL systems. The array antenna characteristics (PSLR, FNBW, null width, and null depth) were then investigated to derive the optimum array configuration. The results showed that the average PSLR and null depth of the proposed array at three frequencies $(88,98$, and $108 \mathrm{MHz}$ ) were greater than those of the UCA configuration by $0.7 \mathrm{~dB}$ and $0.7 \mathrm{~dB}$, respectively. Moreover, the proposed non-uniform array configuration had more suitable beam patterns compared with the conventional UCA. The target detection performance of the proposed array was tested in a certain scenario, and the target was detected with Doppler and range errors of $1 \mathrm{~Hz}$ and $1.2 \mathrm{~km}$, respectively.

This work was supported by a grant-in-aid from Hanwha Systems.

\section{REFERENCES}

[1] S. Paine, D. W. O'Hagan, M. Inggs, C. Schupbach, and U. Boniger, "Evaluating the performance of FM-based PCL radar in the presence of jamming," IEEE Transactions on Aerospace and Electronic Systems, vol. 55, no. 2, pp. 631-643, 2018.

[2] C. R. Berger, B. Demissie, J. Heckenbach, P. Willett, and S. Zhou, "Signal processing for passive radar using OFDM waveforms," IEEE Journal of Selected Topics in Signal Processing, vol. 4, no. 1, pp. 226-238, 2010.

[3] F. Colone, D. Langellotti, and P. Lombardo, "DVB-T signal ambiguity function control for passive radars," IEEE Transactions on Aerospace and Electronic Systems, vol. 50, no. 1, pp. 329-347, 2014.

[4] P. E. Howland, D. Maksimiuk, and G. Reitsma, "FM radio based bistatic radar," IEE Proceedings-Radar, Sonar and Navigation, vol. 152, no. 3, pp. 107-115, 2005.

[5] C. K. Kim, J. S. Lee, J. S. Chae, and S. O. Park, "A modified stripmap SAR processing for vector velocity compensation using the cross-correlation estimation method," Journal of Electromagnetic Engineering and Science, vol. 19, no. 3, pp. 159-165, 2019.

[6] S. G. Ha, J. Cho, J. Lee, B. W. Min, J. Choi, and K. Y. Jung, "Numerical study of estimating the arrival time of UHF signals for partial discharge localization in a power transformer," Journal of Electromagnetic Engineering and Science, vol. 18, no. 2, pp. 94-100, 2018.

[7] J. Wang, H. T. Wang, and Y. Zhao, "Direction finding in frequency-modulated-based passive bistatic radar with a four-element adcock antenna array," IET Radar, Sonar \& Navigation, vol. 5, no. 8, pp. 807-813, 2011.

[8] R. Tao, H. Z. Wu, and T. Shan, "Direct-path suppression by spatial filtering in digital television terrestrial broadcasting-based passive radar," IET Radar, Sonar \& Navigation, vol. 4, no. 6, pp. 791-805, 2010.

[9] M. Malanowski, K. Kulpa, and J. Misiurewicz, "Acceleration estimation for passive coherent location radar," in Proceedings of 2008 IEEE Radar Conference, Rome, Italy, 2008, pp. 1-5.

[10] G. H. Park, D. G. Kim, H. J. Kim, and H. N. Kim, "Maximum-likelihood angle estimator for multi-channel FM-radio-based passive coherent location," IET Radar, Sonar \& Navigation, vol. 12, no. 6, pp. 617-625, 2018.

[11] M. Radmard, S. M. Karbasi, and M. M. Nayebi, "Data fusion in MIMO DVB-T-based passive coherent location," IEEE Transactions on Aerospace and Electronic Systems, vol. 49, no. 3, pp. 1725-1737, 2013.

[12] R. Tharmarasa, M. Subramaniam, N. Nadarajah, T. Kirubarajan, and M. McDonald, "Multitarget passive coher- 
ent location with transmitter-origin and target-altitude uncertainties," IEEE Transactions on Aerospace and Electronic Systems, vol. 48, no. 3, pp. 2530-2550, 2012.

[13] M. Tobias and A. D. Lanterman, "Probability hypothesis density-based multitarget tracking with bistatic range and Doppler observations," IEE Proceedings-Radar, Sonar and Navigation, vol. 152, no. 3, pp. 195-205, 2005.

[14] M. Villano, F. Colone, and P. Lombardo, "Antenna array for passive radar: configuration design and adaptive approaches to disturbance cancellation," International Journal of Antennas and Propagation, vol. 2013, article no. 920639, 2013.

[15] M. Malanowski and K. Kulpa, "Digital beamforming for passive coherent location radar," in Proceedings of 2008

Doyoung Jang

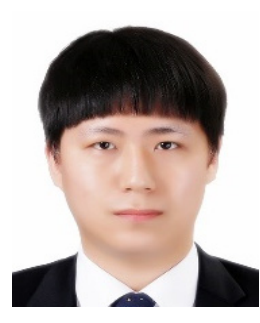

received his B.S. degree in information and telecommunication engineering from Dongyang Mirae University, Seoul, Korea in 2018 and his M.S. degree in electronic and electrical engineering from Hongik University, Seoul, Korea in 2020. He worked as a research engineer in MOASOFT, Seoul, Korea, from 2015 to 2018 . He is currently working toward an Ph.D. degree in electronic and electrical engineering at Hongik University, Seoul, Korea. His research interests include direction finding, passive radars, electromagnetic wave propagation, and electromagnetic environmental effects.

Jun Hur

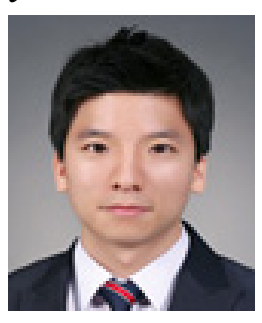

tive beamforming. received his B.S., M.S., and Ph.D. degrees in electronic and electrical engineering from Hongik University, Seoul, Korea in 2014 and 2019, respectively. Since 2019, he has been a postdoctoral researcher at the Metamaterial Electronic Device Research Center of Hongik University. His research interests include global positioning system antennas, antenna arrays, and position optimization of array elements for adap-
IEEE Radar Conference, Rome, Italy, 2008, pp. 1-6.

[16] H. Li, Y. Jiang, Y. Ding, J. Tan, and J. Zhou, "Lowsidelobe pattern synthesis for sparse conformal arrays based on PSO-SOCP optimization," IEEE Access, vol. 6, pp. 77429-77439, 2018.

[17] G. G. Roy, S. Das, P. Chakraborty, and P. N. Suganthan, "Design of non-uniform circular antenna arrays using a modified invasive weed optimization algorithm," IEEE Transactions on Antennas and Propagation, vol. 59, no. 1, pp. 110-118, 2011.

[18] K. K. Yan and Y. Lu, "Sidelobe reduction in array-pattern synthesis using genetic algorithm," IEEE Transactions on Antennas and Propagation, vol. 45, no. 7, pp. 1117-1122, 1997.

Hongsuk Shim

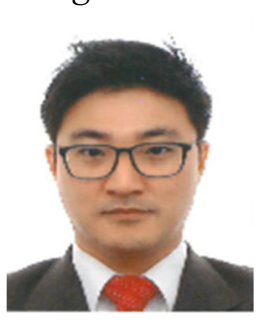

received his B.S. in computer engineering from Chungbuk National University, Cheongju, Korea in 2005 and his M.S. degree in computer engineering from Chungnam National University, Daejeon, Korea in 2008. He is currently working as a researcher for Hanwha Systems, Pangyo, Korea. His research interests include electronic warfare systems, electronic support, electronic attack, and electronic and communications intelligence signal analysis and processing.

Junsik Park

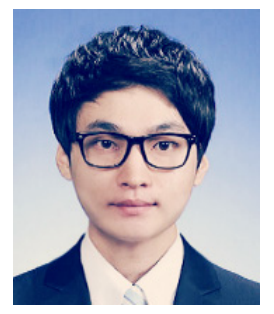

received his B.S. and M.S. degrees in electronic engineering from Chonbuk National University, Jeonju, Korea in 2014 and 2016, respectively. He is currently working as a researcher for Hanwha Systems, Pangyo, Korea. His research interests include electronic warfare systems, electronic support, electronic attack, and military antenna and RF circuits. 


\section{Chihyun Cho}

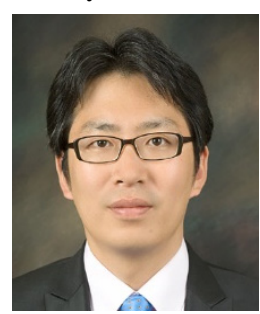

received his B.S., M.S., and Ph.D. degrees in electronic and electrical engineering from Hongik University, Seoul, Korea, in 2004, 2006, and 2009, respectively. From 2009 to 2012, he participated in the development of military communication systems at the Communication R\&D Center, Samsung Thales, Seongnam, Korea. Since 2012, he has been Principal Research Scientist with the Korea Research Institute of Standards and Science, Daejeon, Korea. In 2014, he was a guest researcher at the National Institute of Standards and Technology in Boulder, CO, USA. He also served in the Presidential Advisory Council on Science and Technology in Seoul, Korea in 2016-2017. His current research interests include microwave metrology, time-domain measurements, and standards of communication parameters.

\section{Hosung Choo}

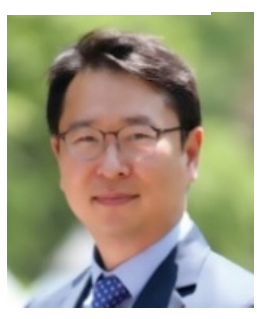

received his B.S. degree in radio science and engineering from Hanyang University in Seoul in 1998 and his M.S. and Ph.D. degrees in electrical and computer engineering from the University of Texas at Austin, in 2000 and 2003, respectively. In September 2003, he joined the school of electronic and electrical engineering, Hongik University, Seoul, Korea, where he is currently a full professor. His principal areas of research are the use of the optimization algorithm in developing antennas and microwave absorbers. His studies include the design of small antennas for wireless communications, reader and tag antennas for RFID, and on-glass and conformal antennas for vehicles and aircraft. 